\title{
UAV BLOCK GEOREFERENCING AND CONTROL BY ON-BOARD GNSS DATA
}

\author{
G. Forlani ${ }^{1, *}$, F. Diotri ${ }^{2}$, U. Morra di Cella ${ }^{2,3}$, R. Roncella ${ }^{1}$ \\ ${ }^{1}$ Dept. of Engineering and Architecture, University of Parma, Parco Area delle Scienze, 181/a, 43124 Parma, Italy - \\ (gianfranco.forlani, riccardo.roncella)@unipr.it \\ 2 Climate Change Unit, Environmental Protection Agency of Valle d'Aosta, Loc. La Maladière, 48, 11020 Saint-Christophe, Italy - \\ fabrizio.diotri@gmail.com, u.morradicella@arpa.vda.it \\ 3 CIMA Foundation, Via A. Magliotto, 2, 17100 Savona, Italy - umberto.morradicella@cimafoundation.org
}

\author{
Commission II, WG 1
}

KEY WORDS: UAV, GNSS-supported block orientation, Bundle Block Adjustment, camera calibration, accuracy

\begin{abstract}
:
Unmanned Aerial Vehicles (UAV) are established platforms for photogrammetric surveys in remote areas. They are lightweight, easy to operate and can allow access to remote sites otherwise difficult (or impossible) to be surveyed with other techniques. Very good accuracy can be obtained also with low-cost UAV platforms as far as a reliable ground control is provided. However, placing ground control points (GCP) in these contexts is time consuming and requires accessibility that, in some cases, can be troublesome. RTKcapable UAV platforms are now available at reasonable costs and can overcome most of these problems, requiring just few (or none at all) GCP and still obtaining accurate results. The paper will present a set of experiments performed in cooperation with ARPA VdA (the Environmental Protection Agency of Valle d'Aosta region, Italy) on a test site in the Italian Alps using a Dji Phantom 4 RTK platform. Its goals are: a) compare accuracies obtainable with different calibration procedures (pre- or on-the-job/self-calibration); b) evaluate the accuracy improvements using different number of GCP when the site allows for it; and c) compare alternative positioning modes for camera projection centres determination, (Network RTK, RTK, Post Processing Kinematic and Single Point Positioning).
\end{abstract}

\section{INTRODUCTION}

\subsection{UAV surveys in mountain areas}

Unmanned Aerial Vehicles (UAV), easy to operate and lightweight enough to be carried on the spot on foot, are established platforms for photogrammetric surveys for agencies in charge of environmental monitoring in mountain regions or remote areas.

As far as some basic aerial photogrammetry rules are correctly employed, very good accuracy can be obtained also with low and middle-cost UAV platform. One of the most critical aspect, considering that often the on-board camera doesn't provide very high resolution and a UAV photogrammetric block can be made up of several hundreds of images, is to provide a reliable ground control. Though providing Ground Control Points (GCP) according to traditional aerial photogrammetry rules, significant systematic deformations show up in the ground coordinates. Many authors addressed, for instance, the so called "dome" or "bowl" effect, e.g. (Jaud et al., 2019), which consists in a local or global systematic deformation of the reconstructed object caused by (random) error propagation between adjacent images and a not accurate orientation estimation procedure, usually due to strong orientation (both interior and exterior) parameters correlation (Oksanen and Sarjakoski, 2006). Providing a good ground control network can limit significantly these effects and improves drastically the final quality of the survey. Consequently, many authors in the recent past investigated thoroughly the influence of GCP number and spatial distribution on the accuracy of the photogrammetric reconstruction, both addressing operational case studies, e.g. (Gindraux et al., 2017), (Tonkin and Midgley, 2016), (James et al., 2017 a) and numerical simulations (James et al., 2017 b).

\footnotetext{
" Corresponding author
}

However, placing and possibly maintaining ground control points in some specific environments (e.g. in mountain regions ${ }^{1}$ or wherever surveying site accessibility is limited) is time consuming and takes a lot of efforts: getting rid of most if not all GCP would be a great improvement. Direct orientation based on integration of Inertial and GNSS data is indeed available to military users and in some custom-made platform (Rehak and Skaloud, 2015), but has not (yet) taken much ground in the UAVs mass-market and in UAV civil photogrammetric applications. To the contrary, RTK-capable UAV platforms are now available at reasonable costs also in mass-market platforms such as the DJI multi-rotor Phantom 4 RTK (Štroner et al., 2020), (Taddia et al., 2020). If the block characteristics are suitable (i.e. basically except in corridor mapping) GNSS observations of Projection Centres (PC) at shooting time are enough to georeference and control the block (Benassi et al., 2017). To send differential corrections to the UAV a local master station is necessary or, alternatively, Network RTK can be used, if internet access is available. If raw data are recorded, Post Processing Kinematic (PPK) brings the potential benefits of more accurate modelling of trajectory (Zhang et al., 2019). An alternative to RTK, where no ground station is necessary, is Precise Point Positioning (Grayson et al, 2018).

Compared to indirect adjustment based on GCP, however, GNSS-assisted block orientation (GNSS-AO) has either strengths as well as weaknesses. Strengths comes from a dense and homogenous block control, as all camera stations are measured. Weaknesses are the risks that inaccurate inner orientation parameters may affect tie points ground coordinates (Zhou et al., 2020). Unlike nadiral blocks with GCP, where with dense enough ground control these errors are to some extent adsorbed by exterior orientation parameters, if block control is limited to camera stations the ground coordinates are not effectively shielded. Accurate camera calibration parameters are 
therefore more important in this case. As far as camera calibration is concerned, three different strategies can be considered (Colomina and Molina, 2014): a) as in traditional aerial photogrammetry, performing a separate pre-calibration procedure via Bundle Block Adjustment (BBA) using an ad-hoc calibration site and acquiring a highly redundant image block (optimally with convergent image configuration and varying the observation distance from the object); b) performing a selfcalibration (or on-the-job) procedure using the same image block considered for the survey; c) using a combination of parameters estimated with procedure a) and b). A pre-calibration was considered by many authors as the best option for obtaining accurate calibration results (Zhou et al., 2018): its stronger image network limits to the minimum the possible correlations between parameters and using an ad-hoc calibration site usually grants a higher level of reliability of the ground control data. However, it is well-known that with most compact cameras currently available in UAV platforms (Rosnell and Honkavaara, 2012) interior orientation (IO) and calibration parameters instabilities can be experienced. In this cases self-calibration can allow estimating more up-to-date parameters. Although in (Daakir et al., 2016) a GCP-free calibration procedure is proposed, selfcalibration with GNSS-supported block orientation should use at least some GCP. In (Hugenholtz et al., 2016) and in (Benassi et al., 2017) the need for at least one GCP has been shown while in (Forlani et al., 2019) pre-calibration performed as good as selfcalibration with one GCP.

Finally, using a combination of parameters, some estimated using pre-calibration procedures, other estimated with an on-the-job or self-calibration approach, might represent the best choice whenever the survey image block is not geometrically robust enough to prevent the insurgence of strong correlations between all the IO and calibration parameters with the Exterior Orientation parameters but, at the same time, invariance of some parameters after the pre-calibration cannot be taken for granted.

\subsection{Paper goals}

Based on the state-of-art and on previous experience from the authors, the paper has three objectives. The first is a comparison between self-calibration and pre-calibration. Out-of-date camera parameters may degrade the accuracy when flying with a precalibrated camera. On the other hand, time or operative constraints in high mountains may force a sub-optimal flight geometry, in turn leading to inaccurate parameter estimation by self-calibration. We are therefore collecting experience on alternatives for calibration to possibly have more choices available, depending on the situation in the field.

The second goal is to evaluate the accuracy improvements using also GCP when the site allows for it.

The third goal is to compare alternative positioning modes for camera projection centres determination, namely: Network RTK (NRTK), RTK, Post Processing Kinematic (PPK) and Single Point Positioning (SPP). From an operational standpoint, all goals are meant to gaining insight on the Dji Phantom 4 RTK platform and its capabilities in one of the standard operating environments of ARPA VdA.

To this aim, an experiment has been set up, with four flights executed over a testfield in Valle d'Aosta.

\section{MATERIALS AND METHODS}

\subsection{Test site description}

The experiment took place in Verrayes $\left(45^{\circ} 45^{\prime} 37^{\prime \prime} \mathrm{N}, 7^{\circ} 32^{\prime}\right.$ $26^{\prime \prime} \mathrm{E}$ ), in a gently undulated sport area, mostly covered by grass, with a height difference of about $20 \mathrm{~m}$. The imaged area is about
380 x $530 \mathrm{~m}$ wide while the actual extent controlled by check points $(\mathrm{CP})$ is smaller: about $330 \times 330 \mathrm{~m}$ wide (see Figure 1). The sport facility is located at about $1020 \mathrm{~m}$ elevation a.s.l. on the main valley (East-West oriented) and has free sky visibility in all directions bar the North.

Nineteen high visibility targets of $50 \times 50 \mathrm{~cm}$ size have been deployed over the area and measured twice with a Leica GS16 in RTK mode with respect to a Geomax Zenith 35 Pro set over a reference point determined in ETRF2000(2008) coordinates with respect to the CORS regional network. The root mean square (RMS) of the differences between the repetitions is $11 \mathrm{~mm}$ for each horizontal coordinate and $13 \mathrm{~mm}$ for elevation.

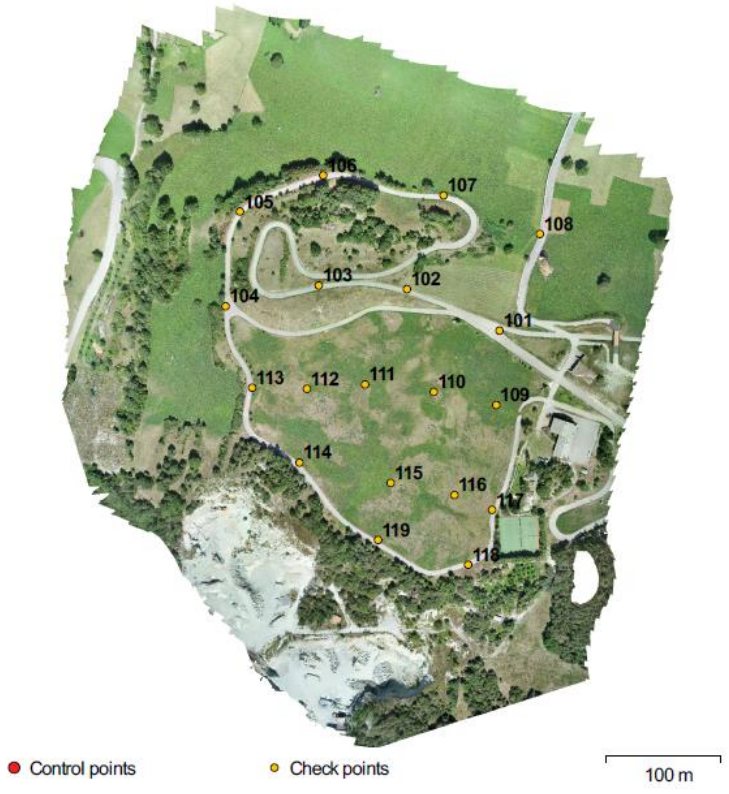

Figure 1. The survey area and the target locations. Most targets act only as check point; some is used as GCP in some tests

The Dji RTK Phantom 4 is equipped with a FC6310R camera with a resolution of $5472 \times 3648$ pixel and a nominal focal length of $8.8 \mathrm{~mm}$; the pixel size is $2.41 \times 2.41 \mu \mathrm{m}$. The Dji kit includes the D-RTK 2 Mobile Station, acting as GNSS base station; however, a Geomax Zenith 35 Pro has been used as additional master station for comparison, according to the third goal of the experiment. Therefore, each survey flight has been independently processed with respect to two ground master stations.

Four flights have been executed. In the first three, the flight plan is the same (see Figure 2), while the positioning mode is different (see Table 1); in the fourth the elevation a.g.l. is about $30 \mathrm{~m}$ higher. The main flight and block characteristics as well as the GNSS data processing modes are summarized in Table 1.

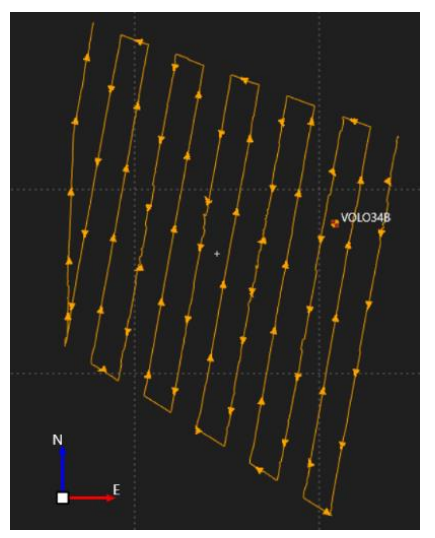

Figure 2. Flight 3 trajectory (same as Flight 1 and 2) 
All nineteen targets could be measured in the first two flights; one went missing between flight 2 and 3 and another two between flight 3 and 4 . In Table 1, fifth row, the minimum and maximum number of manual collimations per target has been reported, showing the high redundancy potential also for automatically selected tie points.

\begin{tabular}{|l|c|c|c|c|}
\hline \multicolumn{1}{|c|}{ Pos. mode } & Flight1 & Flight2 & Flight3 & Flight4 \\
\# images & 324 & RTK & SP & RTK \\
Fwd-lap (\%) & 80 & 80 & 360 & 240 \\
Side-lap (\%) & 70 & 70 & 70 & 80 \\
\# rays per pt & $13-28$ & $19-29$ & $12-28$ & $19-42$ \\
(CP-GCP) & & 83 & 82 & 115 \\
Elev. a.g.l. (m) & 80 & 8.2 & 2.2 & 3.2 \\
GSD (cm) & 2.2 & 2.3 & 16 & 16 \\
Flight time & 16 & 16 & 16 \\
GDOP(m-M) & $2.5-4.4$ & $1.0-1.4$ & $4.2-4.6$ & $2.5-3.3$ \\
CQ 2D avg- & $0.8-2.0$ & $0.4-0.8$ & $0.6-0.8$ & $0.5-1.0$ \\
max (cm) & & & & \\
\hline CQ 1D avg- & $1-2.5$ & $0.7-1.2$ & $0.8-1.4$ & $0.8-1.4$ \\
max (cm) & & & \\
\hline
\end{tabular}

Table 1. Block and GNSS data summary of the four flights

As it can be seen from the last three rows of Table 1, the satellite constellation during flights had varying strength: quite good and stable in Flight 2 and rather weak in Flight 3.

A pre-calibration flight for the $\mathrm{PH} 4$ camera has been executed the day after the four flights (so it is actually a post calibration), on a different location, over two sections at different elevation of a parking area. The calibration flight is made of high overlap regular and cross strips and a set of oblique images taken along a circular flight path with the camera pointing towards the area centre. Fourteen targets have been placed in a regular fashion over the area as GCP; eighteen corners of the parking markings have been used as Check Points and measured with a total station with an estimated accuracy of $0.5 \mathrm{~cm}$ in all coordinates.

\subsection{Block orientation}

The flights have been processed with Photoscan v. 1.2.6. In the various BBA, a standard deviation of $3 \mathrm{~cm}$ has been applied to the PC coordinates, a slightly larger value than those provided in the image Exif (about $1.5 \mathrm{~cm}$ in longitude and latitude and $2 \mathrm{~cm}$ in elevation) as they seem too optimistic compared to the (empirical) precision estimate of the GCP ground survey with the same GNSS configuration and a much better receiver and antenna hardware. As far as GCP are concerned, when used in the adjustment, they have been assigned a standard deviation of $1 \mathrm{~mm}$. The marker and the tie points have been assigned a standard deviation of 0.1 pixel and 1 pixel respectively. This somehow incongruent way to assign measurement precisions depends on the disparity between the amount of information provided by the different types of observations.

The following camera calibration parameters have been considered in the BBA: the inner orientation parameters $c$ (principal distance), $\mathrm{cx}$ and cy (principal point location on the sensor), the radial and tangential distortion coefficients $\mathrm{k} 1, \mathrm{k} 2$, $\mathrm{k} 3, \mathrm{p} 1, \mathrm{p} 2$ and the shape parameters b1, b2.

As far as the tie point residuals after the BBA are concerned, in all cases they are quite stable and very similar for Flight 1 to 3 , at 0.6 pixel, while Flight 4 has slightly larger values, at about 0.8 pixel. In a preliminary orientation stage, aimed basically at providing the tie points, Photoscan has been run on each block with Align parameters set to "High" for Accuracy (i.e. full resolution images are used in feature extraction) and "Disabled" for Pair preselection (i.e. all possible images combinations are considered for tie point matching and stereo-pair relative orientation), using as control information the camera projection centres and in self-calibration mode for camera parameters. In the proper test stages the tie point set has been maintained unchanged and only the camera calibration parameters and the PC coordinates have been changed. When required, the coordinates of selected targets have been fixed to change the point role from CP to GCP.

\subsection{Test overview}

According to the paper goals, the experiment is organized around three tests:

\section{Test 1. Alternatives for calibration in GNSS-AO}

Pre-calibration

- P1: independent calibration flight over a testfield, with GCP

- P2: small calibration block on site, with GCP

- S1: Self-calibration over the survey site, without GCP, on a subset of camera parameters

- S2: Self-calibration over the survey site with one or more GCP, full camera parameter set

Test 2. 3D point accuracy improvements adding GCP

- 1 GCP only vs 4 GCP

- GCP and calibration procedure

Test 3. RTK/NRTK vs PPK

- GNSS processing

- Time interpolation and camera offsets

Test 1: Alternatives for calibration in GNSS-AO: In this stage the objective is to compare pre-calibration and self-calibration. By pre-calibration is intended here the use in the BBA of camera parameters fixed to values obtained by a separate procedure. More precisely, in Case P1 the BBA is executed fixing the camera calibration parameters to the values obtained by an independent, previously executed, calibration flight. In Case P2 a small calibration block, with a few GCP only, made by about 20-40 images was set up in the survey area for pre-calibration. The rationale for this additional effort is to find out whether, taking advantage of the PC positions provided by GNSS, even with a few GCP set up near the master station a quick calibration delivers up-to-date and accurate enough camera parameters to avoid using self-calibration in the main block, where GCP are necessary. This is important in high mountains environment, where the time consuming task of setting targets all around the area conflicts with an often time-constrained mission and is possibly a dangerous activity. The survey area might in fact be far from the ground station, e.g. on a rock face or a glacier. If the placement of the targets can be restricted to a small area nearby, the additional effort can be acceptable in some cases.

In the test, following the findings from a previous experiment (Forlani et al., 2019), rather than setting another small test field, a small subset of images has been extracted from each flight.

More precisely about $10 \%$ of block size has been used in this case (see Table 2). As there are no cross strips in the main blocks, the small blocks are made of 4 to 5 parallel strip extracted in an area where at least 5 GCP are available: in other words, a much less strong block geometry than P1.

\begin{tabular}{|l|c|c|c|}
\hline P2 calibration & Flight1 & Flight2 & Flight4 \\
\hline \# images & 38 & 31 & 36 \\
\hline \# GCP & 5 & 5 & 5 \\
\hline \# rays per GCP & $6-13$ & $9-18$ & $15-24$ \\
\hline
\end{tabular}

Table 2. Case P2 - "on-the-job” calibration blocks features. 
As far as self-calibration is concerned, the procedure consists in a calibration where all or part of the camera parameters are estimated directly in a BBA involving the full image block. In this case three different alternatives can be considered: GCPfree, partial self-calibration BBA and a conventional selfcalibrating BBA. More precisely, in Case S1 all camera PC are used while no GCP is employed; however, the camera principal distance is excluded from the estimated camera parameter set, as the principal distance in GNSS-AO cannot be reliably estimated without GCP, unless oblique images are acquired during the survey. The employed principal distance, for instance, can be estimated with a prior, more reliable, calibration geometry (in this case the principal distance estimated in P1 was considered). A wrong principal distance (probably strongly correlated with other estimated parameters if the block geometry is not strong enough) can lead to significant bias on the computed object point elevation, but it is not claimed here that the principal distance has no influence on the horizontal coordinates, as well. However, as far as horizontal coordinates are the main goal of the survey (and this is true in a number of applications), self-calibration with GNSS-AO can be safely used without GCP, if a reasonably upto-date value of the principal distance is available. Moreover, in those cases where the principal distance is not correct anymore, the user can be alerted spotting high residuals of the PC coordinates.

In Case S2, on the contrary, a full camera parameter set is estimated. Therefore, besides all camera PC, at least a single GCP is necessary. From previous experiences (Benassi et al., 2017), the authors have reasons to believe that this might be enough in some cases, though they are well aware that there is always a reliability problem, i.e. making sure that the coordinates of that only target are accurate, and the position of the single GCP used in the block can affect the results. As our perspective is focussed on surveys under many constraints, where perhaps positioning a single GCP close to the block edge is the only feasible option, it's important to find out how the overall accuracy is affected.

As, under safe conditions, such unique GCP would reasonably be set in the middle of the block, this is precisely what has been done in Case S2 using GCP 111.

\section{Test 2. 3D point accuracy improvements adding GCP}

In Test 2 an analysis of the variability of the accuracy as a function of the point position within the block and of the number of GCP fixed has been performed. Though this would be obviously better tested through numerical simulations, to sense the possible dependence of true GCP accuracy and location in the block, four GCP located along the block edge have been in turn fixed to perform four single-GCP self-calibrations. The average value of the error statistics for those points is also computed.

Fixing just a single GCP in Case $\mathrm{S} 2$ indeed raises the question of whether results depends on its position in the block and on its accuracy. To find out, in addition to using middle-of-block GCP 111, the self-calibrating BBA of Case $\mathrm{S} 2$ has been run also fixing in turn GCP 101, 107, 113 and 117, each roughly located along one of the sides of the block boundary (see Figure 1). All these points have good repeatability in the GNSS double measurement (Section 2.1) and a fair number of rays per point.

In a further step, assuming the survey area being easily accessible, the ground control is increased from a minimal singleGCP configuration to a four-GCP configuration, roughly located at the block corners as recommended in GPS-assisted Aerial Triangulation (Ackermann, 1991). All the above four points have therefore been fixed in a BBA to evaluate the improvement in accuracy. In addition, to highlight the importance of $\mathrm{PC}$ positions in the BBA self-calibration, an additional BBA has been executed without the information from the PC. Finally, 8 GCP, a fair number given the extent of the area, have also been fixed, as it would be in a conventional self-calibrating UAV survey without GNSS-AO.

\section{Test 3. RTK/NRTK vs PPK}

Finally, in Test 3 a comparison is made of the block accuracy obtained using PC coordinates from the Exif data recorded in the images or rather from the time-interpolated positions along the trajectory computed processing the flight GNSS data with respect to the master station. As far as the UAV platform is not equipped with a RTK positioning system, or at the time of the flight a GNSS master station with RTK capabilities is not available, applying a PPK post-processing procedure for PC estimation increase significantly the actual survey accuracy (Chiabrando et al., 2019), (Zhang et al., 2019). Finding whether RTK and PPK positioning provide significantly different results in a UAV image block would also be worth investigating. To this aim, the raw data recorded on board have been downloaded and exported and converted in rinex format and processed in Leica's Infinity environment together with the Geomax ground station data. It turned out that during Flight 1 there was a problem in recording data at the Geomax ground station, so only $3 / 4$ of the PC ( 244 out of 324) could be interpolated from the trajectory. Moreover, processing of GLONASS observations in Leica's Infinity failed in all flights except Flight 2, so only GPS ones could be used. As it could be expected, as baselines are smaller than $500 \mathrm{~m}$, differences below the $\mathrm{mm}$ have been found between the trajectories using Precise or Broadcast Ephemeris. The data acquisition rate for the master station had been set to $5 \mathrm{~Hz}$. Using the time information in the MRK Phantom 4 system log file which provides exposure times and the instantaneous components of the antenna-to-camera projection centre offset in (N, E, Down) system, the PC positions at the shooting time have been computed by linear interpolation.

\section{RESULTS}

In the following sections, the results of the three Test stages summarized in Section 2.3 will be presented.

Notice that results on Flight 3 will be shown only for Test 3 results, as no RTK solution has been collected in such flight (see Table 1).

\subsection{Alternatives for calibration in GNSS-assisted orientation}

In this section the results of the BBA, in terms of the RMS of the residuals on the PC coordinates are first presented, as they measure the goodness of the model fit to the GNSS observations, together with variations in the IO estimated parameters in the four Cases. Finally, to evaluate the accuracy on the ground, the Root Mean Square (RMS) of the discrepancies at the CP are also presented.

Figure 3 shows the RMS of the residuals on the PC coordinates, according to the BBA calibration settings, for Flight 1,2 and 4, obtained with the PC determined by the Dji instrumentation in RTK and NRTK mode. 


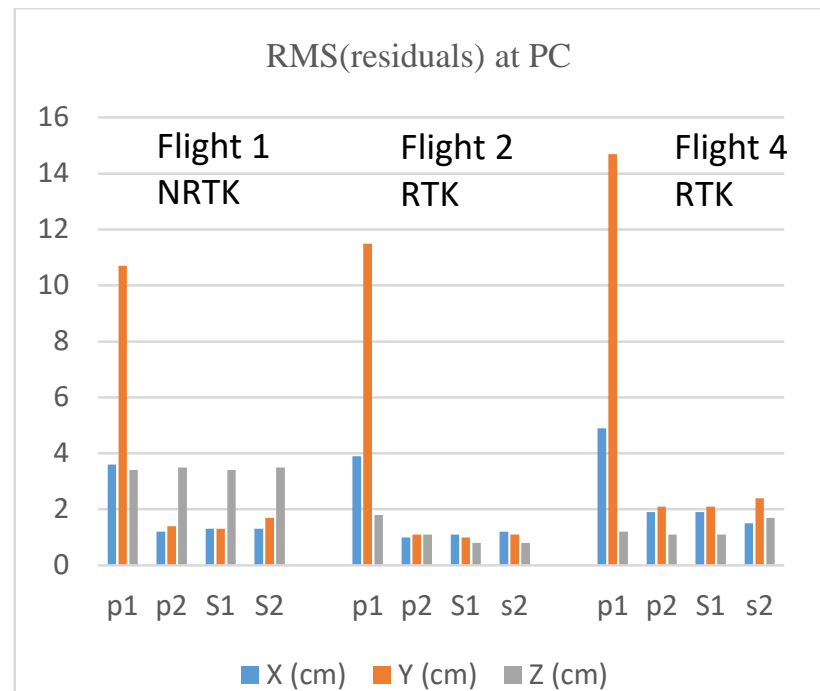

Figure 3. RMS of the residuals on the PC coordinates, according to the BBA calibration settings, for Flight 1,2 and 4.

As it is apparent, the $\mathrm{Y}$ coordinates and, to a less extent, the $\mathrm{X}$ coordinates as well, show anomalous values of the residuals that are significantly larger than the expected precision of RTK. As in Case P1 the pre-calibration parameters are fixed, there are reasons to believe that their values are not representative of the actual camera state. This can be seen from the fact that the residuals in both coordinates drop in all other Cases, where the camera parameters come, in a way or another, from an up-to-date form of calibration. It can also be noticed that the (likely) outdated camera parameters affect the $\mathrm{Z}$ coordinate in different ways in the different flights, despite the fact that at least Flight 1 and 2 have identical flight plans.

A detailed analysis of the changes in the full set of calibration parameters is out of the scope of this paper. However, Table 3 reports the values of the IO parameters applied or estimated in every Case for the three flights. A quick look makes it clear that the principal point position from the pre-calibrated set (Case P1) is clearly not up-to-date by almost 8 pixels in y image coordinate and by about 1 pixel in $\mathrm{x}$ direction. As far as the principal distance is concerned, in Flight 1 and 2 the estimated value is increased roughly of the same amount, while Flight 4, which is at a different elevation, confirm somehow the pre-calibrated value of 3637.5 pixel.

\begin{tabular}{|c|l|c|c|c|}
\hline Case & \multicolumn{1}{|c|}{ Parameter set } & c & cx & cy \\
\hline P1 & Pre-cal flight & 3637.50 & 6.47 & 8.77 \\
\hline P2 & small1 self-c 5GCP & 3638.95 & 7.38 & 16.18 \\
\hline P2 & small2 self-c 5GCP & 3640.14 & 8.12 & 15.31 \\
\hline P2 & small4 self-c 5GCP & 3637.06 & 7.21 & 15.81 \\
\hline S1 & Flight1 self-c c_fix noGCP & 3637.50 & 7.25 & 15.99 \\
\hline S1 & Flight2 self-c c_fix noGCP & 3637.50 & 7.84 & 16.53 \\
\hline S1 & Flight4 self-c c_fix noGCP & 3637.50 & 7.40 & 15.86 \\
\hline S2 & Flight1 self-c 1GCP & 3638.41 & 7.23 & 16.02 \\
\hline S2 & Flight2 self-c 1GCP & 3638.34 & 7.92 & 16.52 \\
\hline S2 & Flight4 self-c 1GCP & 3636.57 & 7.42 & 15.85 \\
\hline
\end{tabular}

Table 3. Estimated principal distance and principal point position for the various cases. "small" refers to the small blocks of the "on-the-job" procedure

Back to Figure 3, a clear anomaly is the pattern of PC residuals in $\mathrm{Z}$ coordinate for Flight 1 , that remain close to $4 \mathrm{~cm}$ in every Case. This fact, that has not yet found a clear explanation, points likely to problems with GNSS data as the reason cannot be simply e.g. a constant error in $\mathrm{Z}$ coordinate. If this were the case, indeed, it would be adsorbed in Case $S 2$ by a change in principal distance. Looking at Table 3, we find indeed an increase by about 1 pixel, that however doesn't solve the inconsistency. This anomaly in $\mathrm{Z}$ residuals will be also remarked in the comparison between the Dji RTK results and the PPK with respect to the Geomax station in Test 3.

Finally, it can be noticed that the residuals in XY coordinates are generally of the same magnitude (slightly larger than $1 \mathrm{~cm}$ ) in Cases P2, S1, S2, except in Flight 4 where they are close to $2 \mathrm{~cm}$ and larger than those in $\mathrm{Z}$.

Looking at P2 estimates in Table 3, it can be noticed that the calibration results are not very stable, though the flights were performed in a few hours (Flight 1 and 2 with a 10' break from one to the other). Admittedly, in each flight the camera undergoes to take off, landing and power off procedures. This dispersion hints at the (already acknowledged) lack of strength of the makeshift calibration block on site; however, also the selfcalibration with the same single GCP produces slight differences among the parameters.

Moving to how the calibration method affects the final reconstruction accuracy, the discrepancies at the check point coordinates are presented in the following. As far as the number of $\mathrm{CP}$ in each test is considered, remember that, as mentioned in Section 2.1, there are 19 CP in Flight 1 and 2, 18 in Flight 3 and 16 only in Flight 4.

Table 4 shows the RMSE of the $\mathrm{CP}$ in each coordinate in GSD units, to make the comparison with other test easier.

The results from Table 4 are fair, though overall a better accuracy could be expected in horizontal coordinates and the $\mathrm{Z}$ coordinate accuracy varies noticeably. In GSD terms, flight 4 , with the largest overlaps and largest elevation a.g.l., has the best results practically under every Case. The Case $\mathrm{S} 2$ procedure, on the other hand, has the best overall results. The large values in $\mathrm{Z}$ for Flight 1 confirm that the problem causing large residuals in the $\mathrm{Z}$ PC coordinates also affects $\mathrm{CP}$ elevations.

\begin{tabular}{|l|c|c|c|c|}
\hline RMSE & Case & X (GSD) & Y (GSD) & Z (GSD) \\
\hline \multirow{4}{*}{ Flight 1 } & $\mathrm{P} 1$ & 1.9 & 1.4 & 2.9 \\
\cline { 2 - 5 } & $\mathrm{P} 2$ & 0.9 & 1.3 & 2.4 \\
\cline { 2 - 5 } & $\mathrm{S} 1$ & 0.7 & 1.3 & 2.4 \\
\cline { 2 - 5 } & $\mathrm{S} 2$ & 0.5 & 0.7 & 2.0 \\
\hline \multirow{4}{*}{ Flight 2 } & $\mathrm{P} 1$ & 1.5 & 2.0 & 1.1 \\
\cline { 2 - 5 } & $\mathrm{P} 2$ & 0.4 & 1.9 & 1.1 \\
\cline { 2 - 5 } & $\mathrm{S} 1$ & 0.3 & 1.9 & 0.9 \\
\cline { 2 - 5 } & $\mathrm{S} 2$ & 0.2 & 1.0 & 1.0 \\
\hline \multirow{4}{*}{ Flight 4 } & $\mathrm{P} 1$ & 1.2 & 1.5 & 1.5 \\
\cline { 2 - 5 } & $\mathrm{P} 2$ & 0.4 & 1.9 & 1.1 \\
\cline { 2 - 5 } & $\mathrm{S} 1$ & 0.2 & 1.3 & 0.7 \\
\cline { 2 - 5 } & $\mathrm{S} 2$ & 0.3 & 0.4 & 0.8 \\
\hline
\end{tabular}

Table 4. Discrepancies at CP in GSD units for the different flights and calibration procedures.

Finally, on average the $\mathrm{Y}$ errors are the largest, almost twice as much as in $\mathrm{X}$ directions. Leaving aside Flight 1, they are 50\% larger than in $\mathrm{Z}$ which is perhaps surprising. Figures 4 and 5 show the RMSE for the horizontal coordinates and the elevation in $\mathrm{cm}$, for all flights and Cases. 


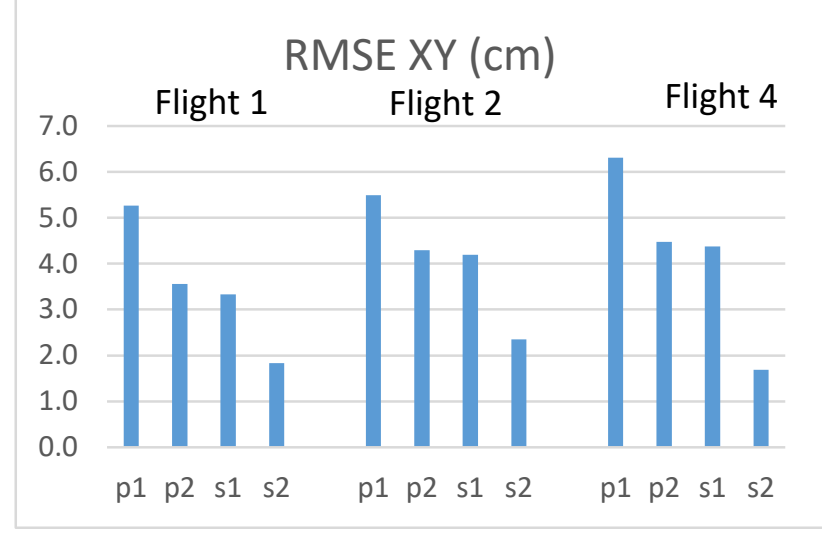

Figure 4. RMSE in horizontal coordinates at CP

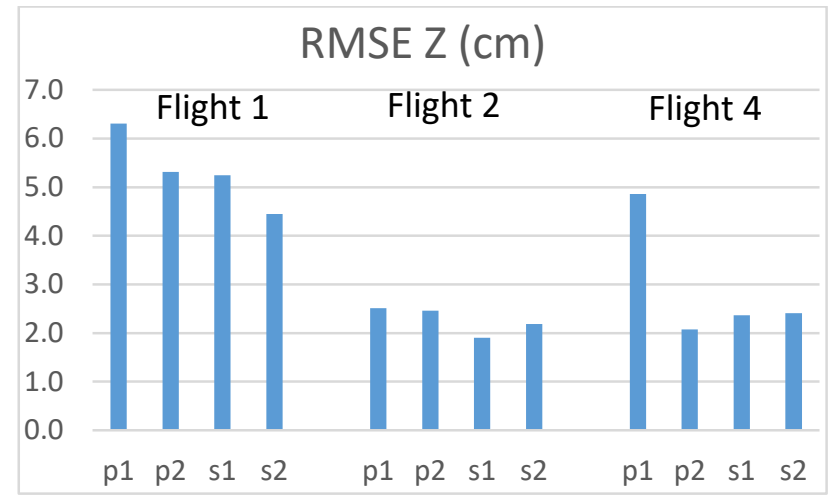

Figure 5. RMSE in elevations at CP

The graphical representations of Figures 4 and 5 allows a clearer picture of the different performance of each calibration procedure. The first remark is that errors in horizontal coordinates has a rather clear and repetitive pattern among the proposed calibration alternatives. Pre-calibration as in P1, where the camera parameters might not be up-to-date might lead to a decrease in accuracy, as it has been the case in Verrayes. It's obviously just a possible outcome: in other projects (Forlani et al., 2019) we found Case P1 on a par with self-calibration in accuracy terms. Case P2 and S1, tough quite different as approach, seem to produce quite similar results. Finally, selfcalibration with one GCP delivers the best accuracy. It should be noticed, however, that the GCP used (GCP 111) is located in the middle of the block, though is not the GCP with the maximum number of ray.

As far as accuracy in elevation is concerned, it's hard to find consistent results. Flight 1, as already noticed, has problems in elevation whatever the calibration method used. In Flight 2 and 4 the results are quite similar for cases $\mathrm{P} 2, \mathrm{~S} 1, \mathrm{~S} 2$ while the effect of out-of-date IO elements is different in $\mathrm{P} 1$ : for some reason, it doesn't much affect Flight 2.

\subsection{D point accuracy improvements adding GCP}

Figures 6 and 7 show the RMSE in horizontal coordinates and elevation of Case S2 for the three flights and each of the four points (blue bars) while the average error is represented by a green one. The RMSE of Case S2 with GCP 111 is shown as a horizontal orange reference line in each case.

It is apparent that the horizontal error measured at the $\mathrm{CP}$, though not in all cases, may vary remarkably, in two cases by about $100 \%$. In elevation (Figure 7) the situation seems more balanced: with cases where the accuracy is the same to other to about $50 \%$ worse. This of course questions the reliability of the single-GCP approach not only due to the GCP accuracy but rather to an overall block stability.

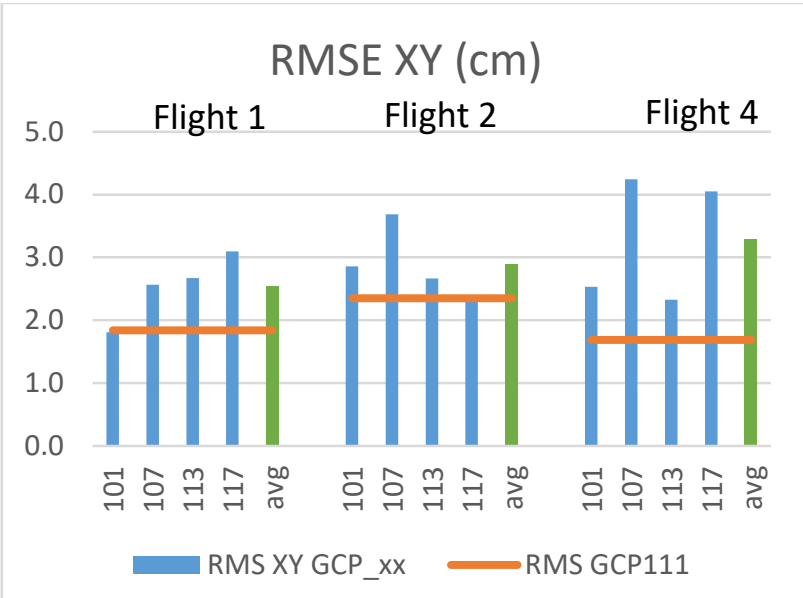

Figure 6. RMSE in horizontal coordinates at $\mathrm{CP}$ in four singleGCP BBA

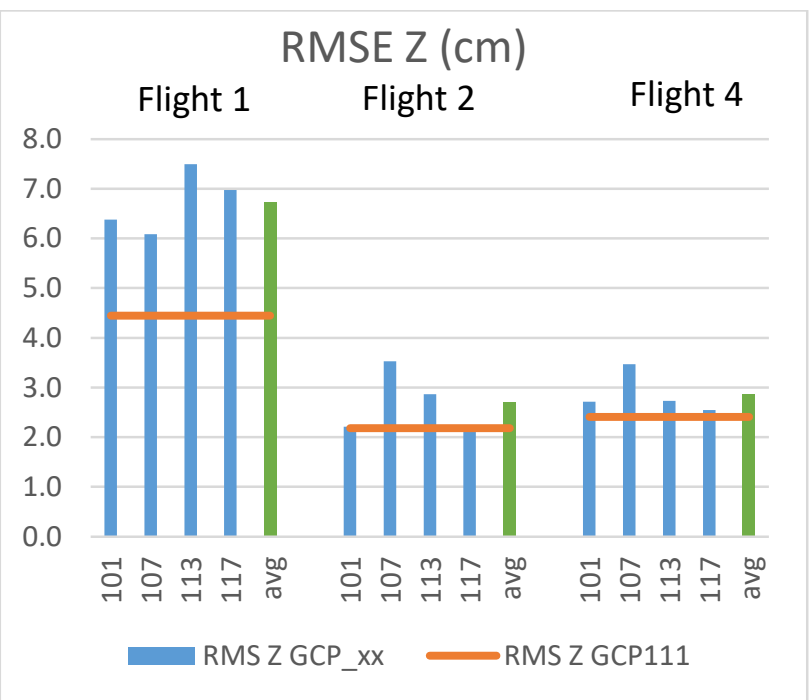

Figure 7. RMSE in elevations at $\mathrm{CP}$ in four single-GCP BBA

Figure 8 and 9 show the RMSE in horizontal coordinates and elevation of a self-calibrating BBA fixing in turn $4 \mathrm{GCP}$ and the PC from RTK data or just 4 and then 8 GCP as in a conventional UAV block.

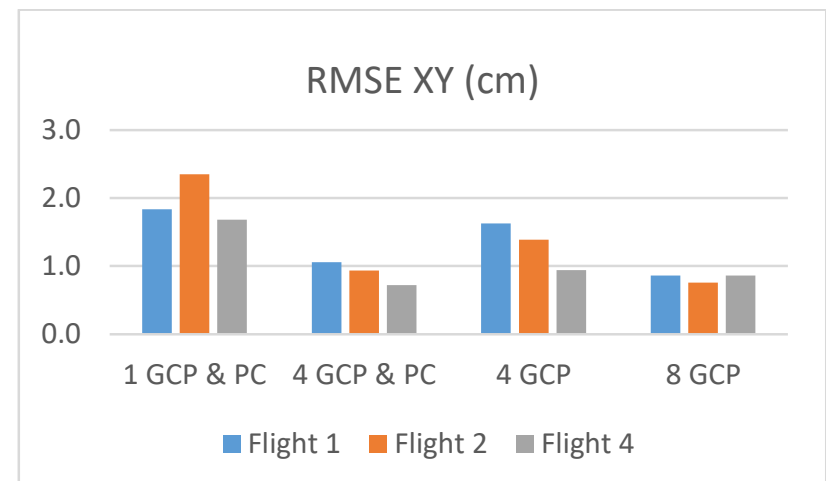

Figure 8. RMSE in horizontal coordinates of $\mathrm{CP}$ with 4-GCP and 8-GCP self-calibrating BBA, with and without GNSS 


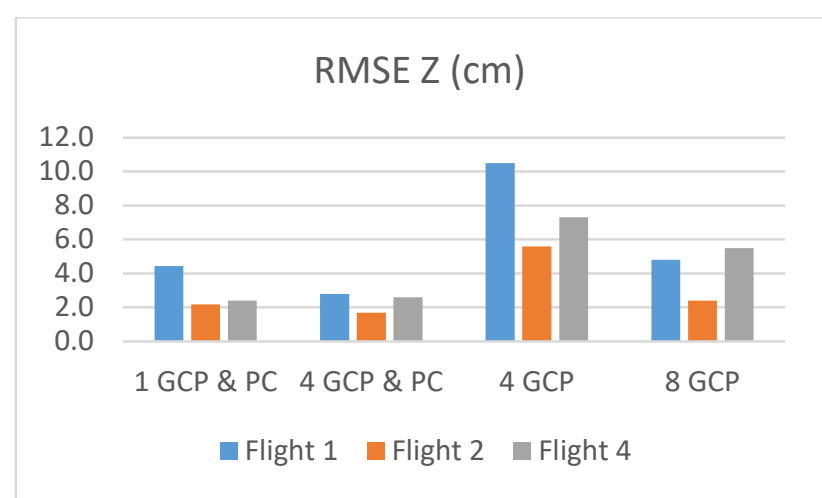

Figure 9. RMSE in elevations of CP with 4-GCP and 8-GCP self-calibrating BBA, with and without GNSS

The results confirm that the horizontal coordinate accuracy is normally still better with appropriate ground control with respect to GNSS-AO with limited control. However, as far as accuracy in elevation is concerned, GNSS-AO looks by far more accurate.

\subsection{SPP/RTK/NRTK vs PPK}

3.3.1 Single Point Positioning SPP: As reported in Table 1, Flight 3 has been performed in SPP mode, i.e. without support of differential corrections in real time. It is confirmed by many studies that SPP accuracy doesn't fit the requirements for UAV photogrammetric block orientation. Flight 3 has been processed according to Case P1, assigning a standard deviation of $3 \mathrm{~m}$ to geotagged $\mathrm{PC}$ positions recorded in the Exif, and to Case S2 (selfcalibration with GCP 111 fixed), progressively decreasing the standard deviation of the PC down to $30 \mathrm{~cm}$ for horizontal coordinates and $120 \mathrm{~cm}$ for elevation, on the basis of the RMS of the residuals. Table 5 shows the RMS of the residuals and the RMSE on the CP for the three BBA.

\begin{tabular}{|l|l|c|c|c|c|c|c|}
\hline & & \multicolumn{3}{|c|}{ RMS(res) PC } & \multicolumn{3}{c|}{ RMSE CP } \\
\hline Case & SD $(\mathrm{cm})$ & X & Y & Z & X & Y & Z \\
\hline P1 & $300 \mathrm{XYZ}$ & 26 & 25 & 59 & 55 & 28 & 310 \\
\hline S2 & $300 \mathrm{XYZ}$ & 42 & 34 & 79 & 32 & 41 & 81 \\
\hline S2 & $30 \mathrm{XY} ; 120 \mathrm{Z}$ & 26 & 20 & 107 & 23 & 28 & 64 \\
\hline
\end{tabular}

Table 5 - Flight 3 . RMS of the residuals of the PC and RMSE on the $\mathrm{CP}$ for Cases P1 and S2, varying the standard deviation of the camera PC. Units in $\mathrm{cm}$.

Figures 10 and 11 show the comparison between RTK and PPK in horizontal coordinates and elevations respectively.

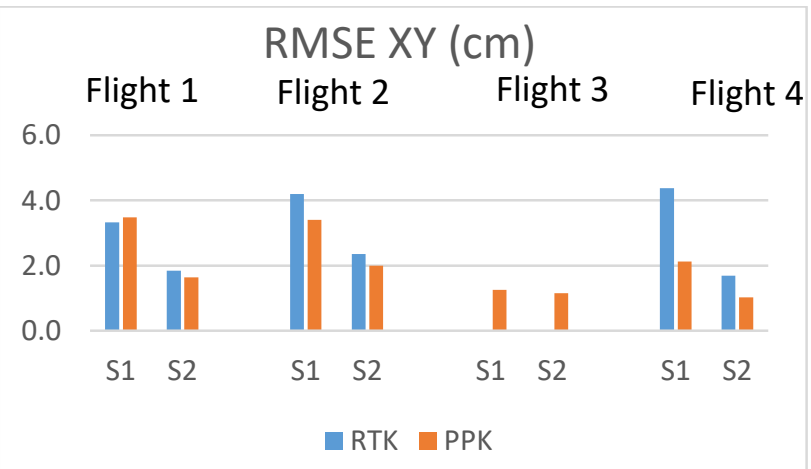

Figure 10. RMSE of the horizontal coordinates in the S1 and S2 Cases for the flight data from RTK and from a PPK solution.

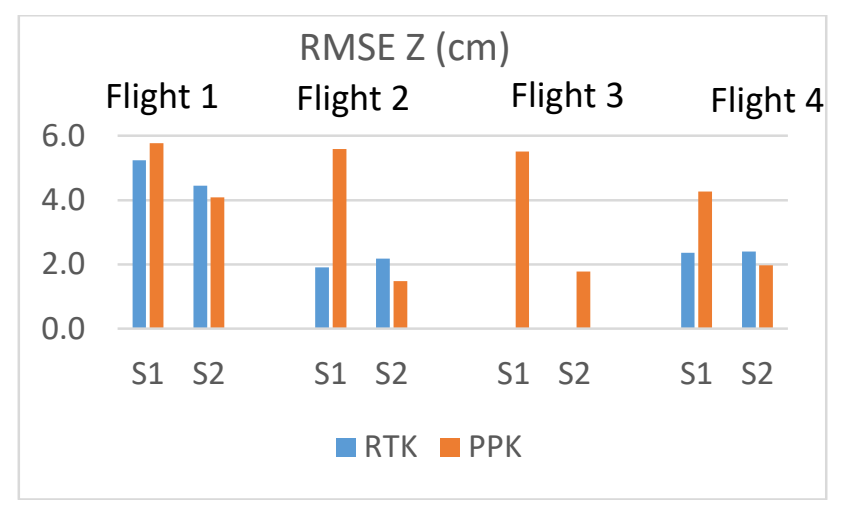

Figure 11. RMSE of the horizontal coordinates in the S1 and S2 Cases for the flight data from RTK and from a PPK solution.

As far as horizontal coordinates are concerned, RTK and PPK provided basically equivalent RMSE in both S1 and S2 Cases, with just Flight 4 showing remarkable differences. In elevation the picture is less clear, with the S2 Case again equivalent but large differences in S1 in favour of the RTK solution.

Figures 12 and 13 show that, at least for Flight 2 and 4, there is in systematic shift between the two solutions, while with Flight 1 the picture is not so clear.

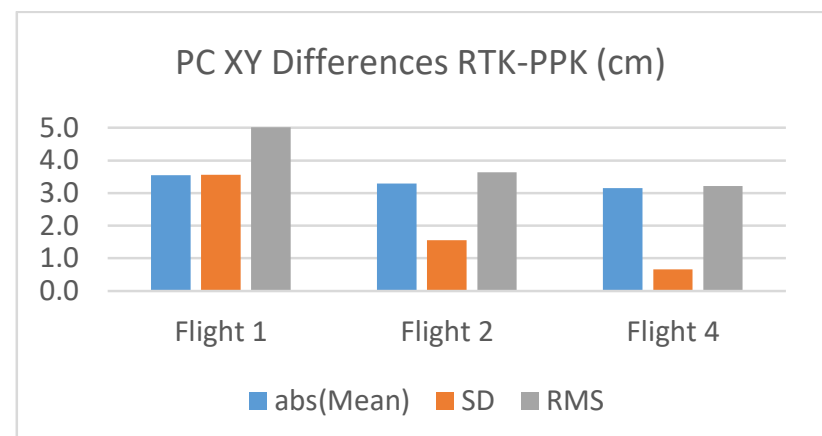

Figure 12. PC XY coordinate differences between the RTK and PPK solutions

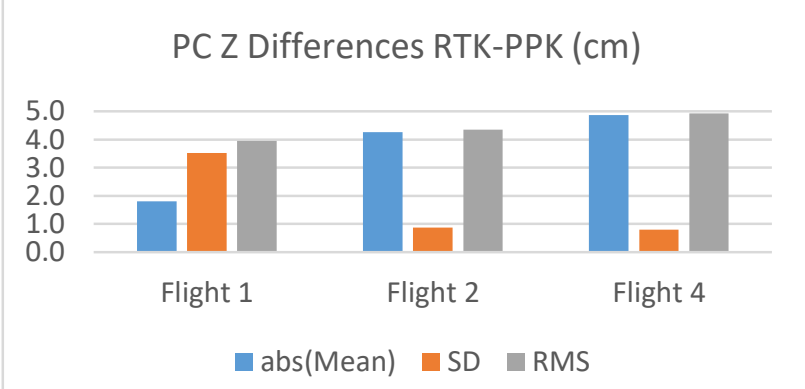

Figure 13. PC Z coordinate differences between the RTK and PPK solutions

\section{CONCLUSIONS}

An analysis of the residuals of projection centres is recommendable to spot model errors or GNSS problems. Indeed, to some extent, outdated calibration parameters can be spotted in the anomalous values of residuals on the PC coordinates. On the other hand, the effect on the ground point coordinates might not necessarily be as dramatic, if the errors at $\mathrm{CP}$ are a reliable indication.

As far as calibration procedures under mission constraints are concerned, the only clear cut indication seems that an 
independent pre-calibration (or a post-calibration, as a matter of fact, in this case) is not reliable and so doesn't deserve the effort it takes to prepare a test field. The test has also shown unstable results, as far as elevation is concerned, in case single-GCP selfcalibration is used. It is therefore advisable using more than one in self-calibration. Other procedures, such as small calibration block on site, have shown instead just slightly worse or equivalent results compared to self-calibration.

Comparison between RTK and PPK provided similar accuracy in horizontal coordinates and better accuracy in elevation for RTK. Improvement of GNSS and trajectory interpolation are still underway, possibly closing the gap, to make sure in case of failure or hardware problems, a NRTK solution might be available. As far as NRTK and RTK perform equivalently, more experience and testing are necessary.

\section{REFERENCES}

Ackermann, F.; Schade, H. 1993. Application of GPS for aerial triangulation. Photogr. Eng. Remote Sens. 59,1625-1632

Benassi, F., Dall'Asta, E., Diotri, F., Forlani, G., Morra di Cella, U., Roncella, R., Santise, M., 2017. Testing Accuracy and Repeatability of UAV Blocks Oriented with GNSS-Supported Aerial Triangulation. Remote Sensing 9, 172.

Chiabrando, F., Giulio Tonolo, F., Lingua, A., 2019. UAV direct georeferencing approach in an emergency mapping context. The 2016 central Italy earthquake case study. Int. Arch. Photogramm. Remote Sens. Spatial Inf. Sci. XLII-2/W13, 247-253.

Colomina, I., Molina, P., 2014. Unmanned aerial systems for photogrammetry and remote sensing: A review. ISPRS Journal of Photogrammetry and Remote Sensing 92, 79-97.

Daakir, M., Pierrot-Deseilligny, M., Bosser, P., Pichard, F., Thom, C., Rabot, Y., 2016. Study of lever-arm effect using embedded photogrammetry and on-board GPS receiver on UAV for metrological mapping purpose and proposal of a free ground measurements calibration procedure, in: ISPRS - International Archives of the Photogrammetry, Remote Sensing and Spatial Information Sciences. Volume XL-3/W4, pp. 65-70.

Forlani, G., Diotri, F., Cella, U.M. di, Roncella, R., 2019. Indirect UAV Strip Georeferencing by On-Board GNSS Data under Poor Satellite Coverage. Remote Sensing 11, 1765.

Gindraux, S., Boesch, R., Farinotti, D., 2017. Accuracy Assessment of Digital Surface Models from Unmanned Aerial Vehicles' Imagery on Glaciers. Remote Sensing 9, 186.

Grayson, B., Penna, N. T., Mills, J. P., \& Grant, D. S. (2018). GPS precise point positioning for UAV photogrammetry. The Photogrammetric Record, 33(164), 427-447.

Hugenholtz, C., Brown, O., Walker, J., Barchyn, T., Nesbit, P., Kucharczyk, M., Myshak, S., 2016. Spatial Accuracy of UAVDerived Orthoimagery and Topography: Comparing Photogrammetric Models Processed with Direct GeoReferencing and Ground Control Points. Geomatica 70, 21-30.

James, M. R., Robson, S., d'Oleire-Oltmanns, S., Niethammer, U., 2017. Optimising UAV topographic surveys processed with structure-from-motion: Ground control quality, quantity and bundle adjustment. Geomorphology 280, 51-66.
James, Mike R., Robson, S., Smith, M.W., 2017. 3-D uncertainty-based topographic change detection with structurefrom-motion photogrammetry: precision maps for ground control and directly georeferenced surveys. Earth Surface Processes and Landforms 42, 1769-1788.

Jaud, M., Passot, S., Allemand, P., Le Dantec, N., Grandjean, P., Delacourt, C., 2019. Suggestions to Limit Geometric Distortions in the Reconstruction of Linear Coastal Landforms by SfM Photogrammetry with PhotoScan ${ }^{\circledR}$ and MicMac ${ }^{\circledR}$ for UAV Surveys with Restricted GCPs Pattern. Drones 3, 2.

Oksanen, J., Sarjakoski, T., 2006. Uncovering the statistical and spatial characteristics of fine toposcale DEM error. International Journal of Geographical Information Science 20, 345-369.

Rehak, M., Skaloud, J., 2015. Fixed-wing micro aerial vehicle for accurate corridor mapping, in: ISPRS Annals of Photogrammetry, Remote Sensing and Spatial Information Sciences. Volume II1/W1, pp. 23-31.

Rosnell, T., Honkavaara, E., 2012. Point Cloud Generation from Aerial Image Data Acquired by a Quadrocopter Type Micro Unmanned Aerial Vehicle and a Digital Still Camera. Sensors 12, 453-480.

Štroner, M., Urban, R., Reindl, T., Seidl, J., Brouček, J., 2020. Evaluation of the Georeferencing Accuracy of a Photogrammetric Model Using a Quadrocopter with Onboard GNSS RTK. Sensors 20, 2318.

Taddia, Y., Stecchi, F., Pellegrinelli, A., 2020. Coastal Mapping Using DJI Phantom 4 RTK in Post-Processing Kinematic Mode. Drones 4, 9.

Tonkin, T.N., Midgley, N.G., 2016. Ground-Control Networks for Image Based Surface Reconstruction: An Investigation of Optimum Survey Designs Using UAV Derived Imagery and Structure-from-Motion Photogrammetry. Remote Sensing 8, 786.

Zhang, H., Aldana-Jague, E., Clapuyt, F., Wilken, F., Vanacker, V., \& Van Oost, K. (2019). Evaluating the potential of postprocessing kinematic (PPK) georeferencing for UAV-based structure-from-motion (SfM) photogrammetry and surface change detection. Earth Surface Dynamics, 7(3), 807-827.

Zhou, Y., Rupnik, E., Faure, P.-H., Pierrot-Deseilligny, M., 2018. GNSS-Assisted Integrated Sensor Orientation with Sensor Pre-Calibration for Accurate Corridor Mapping. Sensors 18, 2783.

Zhou, Y., Rupnik, E., Meynard, C., Thom, C., PierrotDeseilligny, M., 2020. Simulation and Analysis of Photogrammetric UAV Image Blocks-Influence of Camera Calibration Error. Remote Sensing 12, 22. 